\title{
Actinoalloteichus hymeniacidonis sp. nov., an actinomycete isolated from the marine sponge Hymeniacidon perleve
}

\author{
Correspondence \\ Wei Zhang \\ weizhang@dicp.ac.cn \\ Ying Huang \\ huangy@im.ac.cn
}

\author{
Haitao Zhang, ${ }^{1,2}$ Wen Zheng, ${ }^{3}$ Jianyu Huang, ${ }^{1,2}$ Hongli Luo, ${ }^{3}$ Yan Jin, ${ }^{1}$ \\ Wei Zhang, ${ }^{1,4}$ Zhiheng Liu $^{3}$ and Ying Huang ${ }^{3}$ \\ ${ }^{1}$ Marine Bioproducts Engineering Group, Dalian Institute of Chemical Physics, Chinese
Academy of Sciences, Dalian 116023, China \\ ${ }^{2}$ Graduate School of the Chinese Academy of Sciences, Chinese Academy of Sciences, \\ Beijing 100049, China \\ ${ }^{3}$ State Key Laboratory of Microbial Resources, Institute of Microbiology, Chinese Academy of \\ Sciences, Beijing 100080, China \\ ${ }^{4}$ Department of Medical Biotechnology, School of Medicine, Flinders University, Adelaide, \\ SA 5042, Australia
}

\begin{abstract}
A polyphasic study was undertaken to establish the taxonomic status of a Gram-positive, aerobic actinomycete, strain HPA $177^{\top}$, isolated from a marine sponge, Hymeniacidon perleve. The organism formed branching, non-fragmenting vegetative hyphae and produced black pigment. Chemotaxonomic characteristics were consistent with its assignment to the genus Actinoalloteichus. Analysis of the $16 \mathrm{~S}$ rRNA gene sequence showed that strain HPA $177^{\top}$ formed a robust clade with type strains of the genus Actinoalloteichus, but was distinct from them. A number of phenotypic characteristics also readily distinguished strain HPA177 ${ }^{\top}$ from species of the genus Actinoalloteichus with validly published names. On the basis of the above data, it is proposed that strain HPA $177^{\top}$ represents a novel species, Actinoalloteichus hymeniacidonis sp. nov. The type strain of Actinoalloteichus hymeniacidonis is HPA177 ${ }^{\top}$ (=CGMCC $4.2500^{\top}=\mathrm{JCM} 13436^{\top}$ ).
\end{abstract}

The genus Actinoalloteichus was established by Tamura et al. (2000) after several initial taxonomic studies on its phenotypic and genotypic properties by Liu et al. (1984), Itoh et al. (1987) and Tamura \& Hatano (1998). The genus currently comprises only two species, Actinoalloteichus cyanogriseus (Tamura et al., 2000) and Actinoalloteichus spitiensis (Singla et al., 2005). During the investigation of actinomycete diversity of an intertidal marine sponge, Hymeniacidon perleve, a novel actinomycete, strain HPA177 ${ }^{\mathrm{T}}$, was isolated. The aim of the present study was to determine the taxonomic status of this strain using a polyphasic approach.

Strain HPA $177^{\mathrm{T}}$ was isolated by the following procedure. The marine sponge was collected at the inter-tidal beach of Dalian, on the Chinese Yellow Sea, located in northern China $\left(38^{\circ} 52^{\prime} \mathrm{N} 121^{\circ} 41^{\prime} \mathrm{E}\right)$. Freshly collected sponge specimens were rinsed five times in sterile seawater to remove transient and loosely attached bacteria and then thoroughly homogenized in a sterile mortar. A 10-fold

Abbreviation: $A_{2} p m$, diaminopimelic acid.

The GenBank/EMBL/DDBJ accession number for the $16 \mathrm{~S}$ rRNA gene sequence of strain HPA $177^{\top}$ is DQ144222. dilution series of sponge homogenate was made and plated in triplicate on modified arginine/glycerol agar (ISP medium 5) plates (6 ml $100 \%$ glycerol, $1 \mathrm{~g}$ arginine, $1 \mathrm{~g}$ $\mathrm{K}_{2} \mathrm{HPO}_{4}, 0.5 \mathrm{~g} \mathrm{MgSO}_{4}, 18 \mathrm{~g}$ agar and $1 \mathrm{l}$ natural seawater). After incubating the plates at $28{ }^{\circ} \mathrm{C}$ for 4 weeks, an isolate, designated HPA $177^{\mathrm{T}}$, was picked and subcultured on TSA plates (Difco) until a pure culture was obtained.

The arrangement of hyphae and spore chains of strain HPA $177^{\mathrm{T}}$ were observed on yeast extract/malt extract agar (ISP medium 2) and oatmeal agar (ISP medium 3) after 2-3 weeks at $28^{\circ} \mathrm{C}$ using light and scanning electron microscopes. Cultural characteristics were observed on a number of standard media (Table 1) after 2 weeks at $28^{\circ} \mathrm{C}$. The strain was examined further for a range of physiological properties using established procedures described by Gordon et al. (1974) and Yokota et al. (1993). Biomass for chemical studies was prepared by growing the strain in shake flasks of TSB at $28^{\circ} \mathrm{C}$ for 7 days. Cells were harvested by centrifugation, washed with distilled water and freezedried. The isomers of diaminopimelic acid $\left(\mathrm{A}_{2} \mathrm{pm}\right)$ and whole-organism sugars were analysed according to the procedures developed by Hasegawa et al. (1983) and Lechevalier 
Table 1. Growth and cultural characteristics of strain HPA $177^{\top}$

\begin{tabular}{|lllll|}
\hline Agar medium & Growth & \multicolumn{1}{c|}{ Aerial mycelium } & Substrate mycelium & Diffusible pigment \\
\hline Yeast extract/malt extract (ISP 2) & Abundant & Brownish-purplish-grey; abundant & Brownish-black & Black \\
Oatmeal (ISP 3) & Abundant & Pale grey; abundant & Grey & Smoky grey \\
Inorganic salts/starch (ISP 4) & Poor & Colourless; poor & Pale grey & None \\
Glycerol/asparagine (ISP 5) & Poor & Pale greyish-white; moderate & Light olive grey & Light purplish-grey \\
Peptone/yeast extract/iron (ISP 6) & Moderate & Greyish-white; moderate & Greyish-brown & Black \\
Tyrosine (ISP 7) & Poor & Greyish-white; poor & Light greyish-brown & Greyish-brown \\
Modified Bennett's & Abundant & Brownish-grey; abundant & Olive black & Black \\
\hline
\end{tabular}

\& Lechevalier (1980). Polar lipids were examined by twodimensional TLC and identified using the method of Minnikin et al. (1984). Menaquinones were extracted from freeze-dried biomass and purified according to Collins (1985); the purified preparation was analysed using an HPLC procedure (Wu et al., 1989). Mycolic acids were checked by the acid methanolysis method as described previously (Minnikin et al., 1980). Fatty acids were extracted, methylated and analysed by GC using the standard Sherlock MIDI (Microbial Identification) system (Sasser, 1990; Kämpfer \& Kroppenstedt, 1996).

The morphological characteristics of strain HPA $177^{\mathrm{T}}$ were similar to those of $A$. cyanogriseus, the type species of the genus. It formed grey to black, branched vegetative hyphae that tended to fragment after 3 weeks of cultivation, aerial hyphae with straight spore chains (Fig. 1) and a greyish aerial spore mass on several standard media (Table 1). The assignment of strain $\mathrm{HPA}_{177^{\mathrm{T}}}$ to the genus Actinoalloteichus is also supported by its chemotaxonomic profile. This strain contained meso- $\mathrm{A}_{2} \mathrm{pm}$ as the cell wall diamino acid, galactose, glucose, mannose and ribose as the diagnostic sugars in whole-organism hydrolysates and tetrahydrogenated menaquinone with nine isoprene units $\left[\mathrm{MK}-9\left(\mathrm{H}_{4}\right)\right](64 \%)$ as the

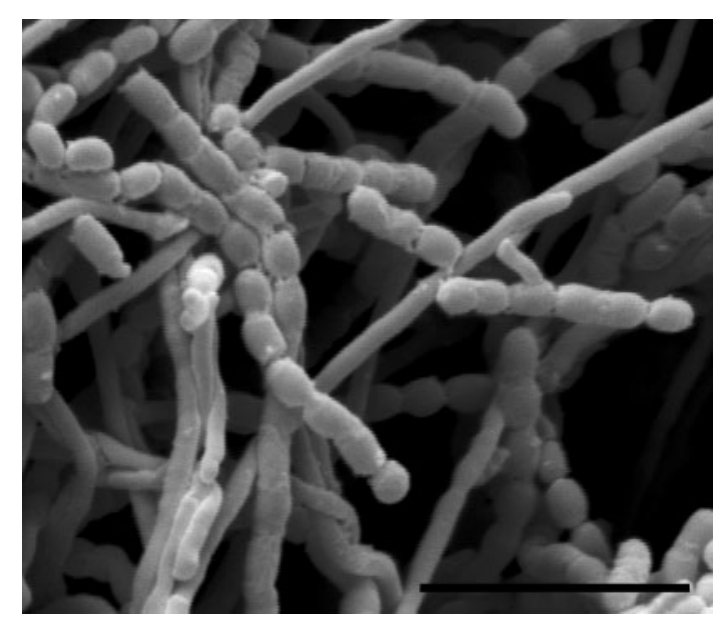

Fig. 1. Scanning electron micrograph of strain HPA $177^{\top}$ grown on yeast extract/malt extract agar for 14 days at $28^{\circ} \mathrm{C}$. Bar, $5 \mu \mathrm{m}$. predominant isoprenoid quinone, with smaller amounts of MK-9 $\left(\mathrm{H}_{6}\right)(23 \%)$ and MK-9 $\left(\mathrm{H}_{8}\right)(12 \%)$. It also contained phosphatidylethanolamine, phosphatidylglycerol, phosphatidylinositol, phosphatidylinositol mannoside and some phospholipids of unknown structure containing glucosamine as diagnostic polar lipids, but lacked mycolic acids (data not shown). The predominant cellular fatty acids were $15: 0$ anteiso $(20 \%), 17: 1 \omega 8 c(19 \%), 16: 0$ iso $(16 \%)$ and $17: 0(11 \%)$. Strain HPA $177^{\mathrm{T}}$ differed significantly from $A$. cyanogriseus (Tamura et al., 2000) and A. spitiensis (Singla et al., 2005) in having larger amounts of $17: 1 \omega 8 c$ and $17: 0$. The complete fatty acid profile and differences from those of A. cyanogriseus and A. spitiensis are shown in Table 2.

Table 2. Chemotaxonomic characteristics that distinguish strain HPA $177^{\top}$ from $A$. cyanogriseus IFO $14455^{\top}$ and $A$. spitiensis DSM $44848^{\top}$

Strains: 1, HPA $177^{\mathrm{T}}$; 2, A. cyanogriseus IFO $14455^{\mathrm{T}}$ (data from Tamura et al., 2000); 3, A. spitiensis DSM $44848^{\mathrm{T}}$ (data from Singla et al., 2005). Values less than $2 \%$ are not shown. -, Not detected.

\begin{tabular}{|c|c|c|c|}
\hline Characteristic & 1 & 2 & 3 \\
\hline \multicolumn{4}{|c|}{ Cellular fatty acids (\%) } \\
\hline $14: 0$ iso & 4 & 5 & 6 \\
\hline $15: 0$ iso & 6 & 15 & 17 \\
\hline $15: 0$ anteiso & 20 & 10 & 7 \\
\hline $15: 0$ & 6 & - & - \\
\hline $16: 1$ iso & - & 8 & 6 \\
\hline $16: 1$ & - & 2 & 2 \\
\hline $16: 0$ iso $G$ & 6 & - & - \\
\hline $16: 0$ iso & 16 & 19 & 33 \\
\hline $16: 0$ & - & 2 & 2 \\
\hline $17: 0$ iso & - & 3 & 3 \\
\hline $17: 0$ anteiso & 4 & 20 & 8 \\
\hline $17: 1 \omega 8 c$ & 19 & - & - \\
\hline $17: 0$ & 11 & - & - \\
\hline \multicolumn{4}{|c|}{ Menaquinones (\%) } \\
\hline $\mathrm{MK}-8\left(\mathrm{H}_{4}\right)$ & - & 10 & 5 \\
\hline MK-9 $\left(\mathrm{H}_{2}\right)$ & - & 9 & - \\
\hline MK-9 $\left(\mathrm{H}_{4}\right)$ & 64 & 75 & 82 \\
\hline MK-9( $\left.\mathrm{H}_{6}\right)$ & 23 & 2 & 2 \\
\hline MK-9 $\left(\mathrm{H}_{8}\right)$ & 12 & - & - \\
\hline MK-10 $\left(\mathrm{H}_{4}\right)$ & - & 3 & 9 \\
\hline
\end{tabular}




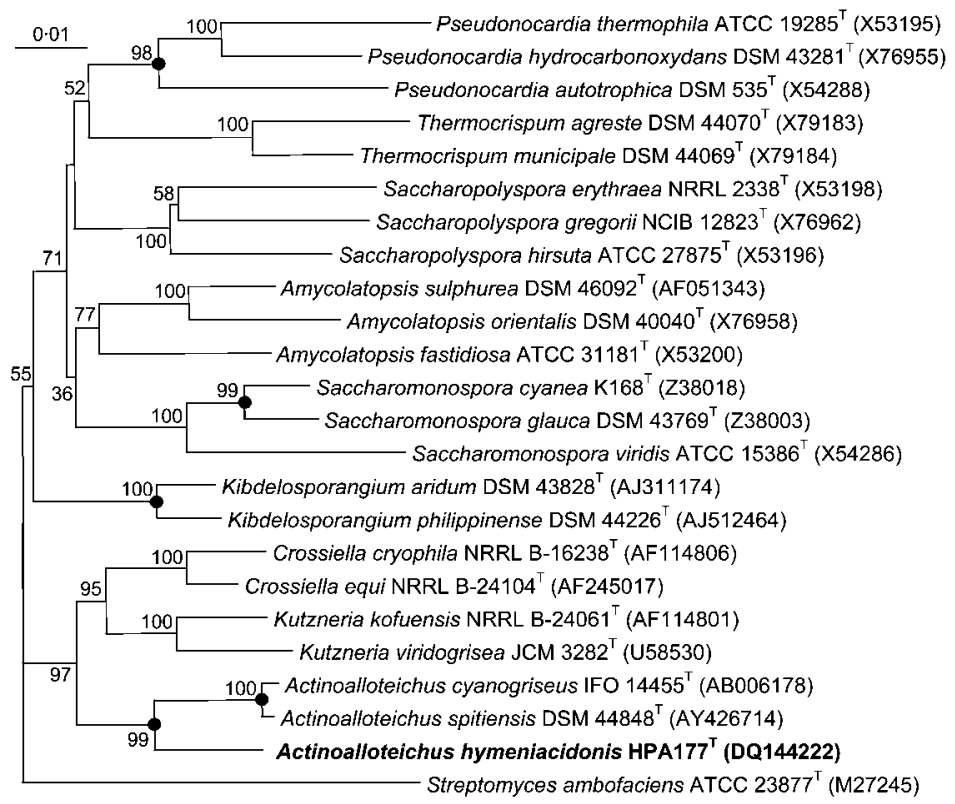

Fig. 2. Neighbour-joining tree showing the phylogenetic position of strain HPA $177^{\top}$ and related taxa based on 16S rRNA gene sequences. Streptomyces ambofaciens ATCC $23877^{\top}$ was used as the root organism. Numbers at nodes represent levels of bootstrap support, based on neighbour-joining analyses of 1000 resampled datasets. Solid circles represent clades that were also recovered in the maximum-parsimony tree. Bar, 0.01 substitutions per nucleotide position.

For 16S rRNA gene sequencing, genomic DNA of HPA177 ${ }^{\mathrm{T}}$ was extracted as described previously by Lee et al. (2003) and PCR amplification and sequencing of the $16 \mathrm{~S}$ rRNA gene were performed as described by Webster \& Hill (2001). The resultant sequence (1470 bp) was compared with those

Table 3. Differential physiological characteristics of strain

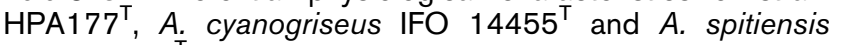
DSM $44848^{\top}$

Strains: 1, HPA $177^{\mathrm{T}}$; 2, A. cyanogriseus IFO $14455^{\mathrm{T}} ; 3$, A. spitiensis DSM $44848^{\mathrm{T}} .++$, Good; + , moderate; $+/-$, poor; - , none.

\begin{tabular}{|c|c|c|c|}
\hline Characteristic & 1 & 2 & 3 \\
\hline \multicolumn{4}{|l|}{ Utilization of: } \\
\hline Arabinose & - & $+1-$ & - \\
\hline Fructose & + & - & - \\
\hline Glucose & ++ & ++ & - \\
\hline Maltose & ++ & ++ & - \\
\hline Mannitol & ++ & + & + \\
\hline Mannose & ++ & + & - \\
\hline Raffinose & - & - & + \\
\hline Xylose & + & + & - \\
\hline Rhamnose & + & ++ & - \\
\hline Sucrose & + & $+1-$ & + \\
\hline Sorbitol & + & + & - \\
\hline Sodium citrate & + & - & + \\
\hline Sodium succinate & - & - & + \\
\hline Decomposition of casein & + & - & + \\
\hline Hydrolysis of starch & + & $+1-$ & + \\
\hline Resistance to methyl violet & + & - & - \\
\hline \multicolumn{4}{|l|}{ Pigmentation in: } \\
\hline ISP 1 & + & - & - \\
\hline ISP 6 & + & + & - \\
\hline
\end{tabular}

deposited in public databases. Phylogenetic analysis was performed using the neighbour-joining (Saitou \& Nei, 1987) and maximum-parsimony (Fitch, 1972) methods in the PHYLIP package version 3.6 (Felsenstein, 1985), after multiple alignment of data by CLUSTAL $\mathrm{x}$ version 1.8 (Thompson et al., 1997). Evolutionary distances were calculated according to the two-parameter model of Kimura (1980). Tree topologies were evaluated by bootstrap analyses (Felsenstein, 1985) based on 1000 replications. It is clear from Fig. 2 that strain $\mathrm{HPA} 177^{\mathrm{T}}$ forms a distinct lineage within the evolutionary radiation encompassed by the genus Actinoalloteichus, supported by both tree-making algorithms and by a $99 \%$ bootstrap value recorded using the neighbour-joining method. Sequence similarity calculations after neighbour-joining analysis indicated that the isolate shared relatively low similarities with its closest relatives, A. spitiensis DSM $44848^{\mathrm{T}}(97 \cdot 1 \%)$ and A. cyanogriseus IFO $14455^{\mathrm{T}}(96 \cdot 7 \%)$. Sequence similarities to all other species of related genera with validly published names were lower than $95 \%$. Strain HPA177 ${ }^{\mathrm{T}}$ could also be distinguished from members of the genus Actinoalloteichus using a combination of phenotypic properties (Table 3 ).

It is evident from the genotypic and phenotypic data that strain HPA177 ${ }^{\mathrm{T}}$ should be classified as a representative of a novel Actinoalloteichus species; the name Actinoalloteichus hymeniacidonis sp. nov. is proposed.

\section{Description of Actinoalloteichus hymeniacidonis sp. nov.}

Actinoalloteichus hymeniacidonis (hy.me.ni.a.ci'do.nis. N.L. gen. n. hymeniacidonis of Hymeniacidon, the generic name of the marine sponge Hymeniacidon perleve, the source of the type strain). 
Gram-positive, strictly aerobic actinomycete with branching hyphae. Non-fragmenting substrate mycelia are present within 3 weeks of cultivation. Aerial mycelia with straight spore $(0.6 \times 0.8 \mu \mathrm{m})$ chains aggregate. Grows well on yeast extract $/$ malt extract agar and oatmeal agar at $20-37^{\circ} \mathrm{C}$. A black soluble pigment is produced on yeast extract $/ \mathrm{malt}$ extract agar and peptone/yeast extract/iron agar. Decomposition of urea, growth in Sabouraud's dextrose broth and MacConkey agar, hydrolysis of aesculin and hippurate, utilization of calcium malate, sodium oxalate and sodium succinate and reduction of nitrate are all negative. Fructose, glucose, maltose, mannose, mannitol, rhamnose, sucrose, sorbitol and xylose are utilized as sole carbon sources, but arabinose, inositol and raffinose are not. Grows weakly at $15^{\circ} \mathrm{C}$ and does not grow at $45^{\circ} \mathrm{C}$. The cell wall chemotype is III. The major menaquinone is MK-9 $\left(\mathrm{H}_{4}\right)$; small amounts of MK-9 $\left(\mathrm{H}_{6}\right)$ and MK-9 $\left(\mathrm{H}_{8}\right)$ are also present. The phospholipid profile comprises mainly phosphatidylethanolamine, phosphatidylglycerol, phosphatidylinositol and phosphatidylinositol mannoside. Major fatty acids are 15:0 anteiso (20\%), $17: 1 \omega 8 c(19 \%), 16: 0$ iso (16\%) and $17: 0(11 \%)$.

The type strain is $\mathrm{HPA}_{177^{\mathrm{T}}}\left(=\mathrm{CGMCC} 4.2500^{\mathrm{T}}=\mathrm{JCM}\right.$ $13436^{\mathrm{T}}$ ), isolated from the marine sponge Hymeniacidon perleve in Dalian, China.

\section{Acknowledgements}

This work was supported by the ' 100 Talent Project' of the Chinese Academy of Sciences, 'Innovation Fund' from Dalian Institute of Chemical Physics and '973 Hi-Tech Research and Development Program of China' (2003CB716001). The authors are grateful to Professor Jin-He Li (Qingdao Institute of Oceanology, Chinese Academy of Sciences) for identification of the sponge and to Professor J. P. Euzéby (Toulouse) for his invaluable help with the nomenclature of the novel species.

\section{References}

Collins, M. D. (1985). Isoprenoid quinone analyses in bacterial classification and identification. In Chemical Methods in Bacterial Systematics, pp. 267-284. Edited by M. Goodfellow \& D. E. Minnikin. London: Academic Press.

Felsenstein, J. (1985). Confidence limits on phylogenies: an approach using the bootstrap. Evolution 39, 783-791.

Fitch, W. M. (1971). Toward defining the course of evolution: minimum change for a specific tree topology. Syst Zool 20, 406-416.

Gordon, R. E., Barnett, D. A., Handerhan, J. E. \& Pang, C. H.-N. (1974). Nocardia coeliaca, Nocardia autotrophica, and the nocardin strain. Int J Syst Bacteriol 24, 54-63.

Hasegawa, T., Takizawa, M. \& Tanida, S. (1983). A rapid analysis for chemical grouping of aerobic actinomycetes. J Gen Appl Microbiol 29, 319-322.
Itoh, T., Kudo, T. \& Seino, A. (1987). Chemotaxonomic studies on new genera of actinomycetes proposed in Chinese papers. Actinomycetologica 1, 43-59.

Kämpfer, P. \& Kroppenstedt, R. M. (1996). Numerical analysis of fatty acid patterns of coryneform bacteria and related taxa. Can J Microbiol 42, 989-1005.

Kimura, M. (1980). A simple method for estimating evolutionary rates of base substitutions through comparative studies of nucleotide sequences. J Mol Evol 16, 111-120.

Lechevalier, M. P. \& Lechevalier, H. A. (1980). The chemotaxonomy of actinomycetes. In Actinomycete Taxonomy, Special Publication 6, pp. 277-284. Arlington, VA: Society for Industrial Microbiology.

Lee, Y. K., Kim, H. W., Liu, C. L. \& Lee, H. K. (2003). A simple method for DNA extraction from marine bacteria that produce extracellular materials. J Microbiol Methods 52, 245-250.

Liu, Z., Zhang, Y. \& Yan, X. (1984). A new genus of the order Actinomycetales. Acta Microbiol Sin 24, 295-298.

Minnikin, D. E., Hutchinson, I. G., Caldicott, A. B. \& Goodfellow, M. (1980). Thin-layer chromatography of methanolysates of mycolic acid-containing bacteria. J Chromatogr 188, 221-233.

Minnikin, D. E., O’Donnell, A. G., Goodfellow, M., Alderson, G., Athalye, M., Schaal, K. \& Parlett, J. H. (1984). An integrated procedure for the extraction of bacterial isoprenoid quinones and polar lipids. J Microbiol Methods 2, 233-241.

Saitou, N. \& Nei, M. (1987). The neighbor-joining method: a new method for reconstructing phylogenetic trees. Mol Biol Evol 4, 406-425.

Sasser, M. (1990). Identification of bacteria by gas chromatography of cellular fatty acids. Technical Note 101. Newark, DE: MIDI.

Singla, A. K., Mayilraj, S., Kudo, T., Krishnamurthi, S., Prasad, G. S. \& Vohra, R. M. (2005). Actinoalloteichus spitiensis sp. nov., a novel actinobacterium isolated from a cold desert of the Indian Himalayas. Int J Syst Evol Microbiol 55, 2561-2564.

Tamura, T. \& Hatano, K. (1998). Phylogenetic analyses on the strains belonging to invalidated genera of the order Actinomycetales. Actinomycetologica 12, 15-28.

Tamura, T., Liu, Z., Zhang, Y. \& Hatano, K. (2000). Actinoalloteichus cyanogriseus gen. nov., sp. nov. Int J Syst Evol Microbiol 50, $1435-1440$

Thompson, J. D., Gibson, T. J., Plewniak, F., Jeanmougin, F. \& Higgins, D. G. (1997). The CLUSTAL_X windows interface: flexible strategies for multiple sequence alignment aided by quality analysis tools. Nucleic Acids Res 25, 4876-4882.

Webster, N. S. \& Hill, R. T. (2001). The culturable microbial community of the Great Barrier Reef sponge Rhopaloeides odorabile is dominated by an $\alpha$-proteobacterium. Mar Biol 138, 843-851.

Wu, C., Lu, X., Qin, M., Wang, Y. \& Ruan, J. (1989). Analysis of menaquinone compound in microbial cells by HPLC. Microbiology [English translation of Microbiology (Beijing)] 16, 176-178.

Yokota, A., Tamura, T., Hasegawa, T. \& Huang, L. H. (1993). Catenuloplanes japonicus gen. nov., sp. nov., nom. rev., a new genus of the order Actinomycetales. Int J Syst Bacteriol 43, 805-812. 